\title{
The collineations which act as addition and multiplication on points in a certain class of projective Klingenberg planes
}

\author{
Basri Celik* and Abdurrahman Dayioglu
}

\section{"Correspondence:}

basri@uludag.edu.tr

Department of Mathematics,

Faculty of Arts and Science, Uludag

University, Gorukle, Bursa, Turkiye

\begin{abstract}
Let $P K_{2}(\mathbf{Q}(\varepsilon))$ be the projective Klingenberg plane coordinated by the dual quaternion ring $\mathbf{Q}(\varepsilon)=\mathbf{Q}+\mathbf{Q} \varepsilon=\{x+y \varepsilon \mid x, y \in \mathbf{Q}\}$ where $\mathbf{Q}$ is any quaternion ring. In this paper, we determine the addition and multiplication of the points on the line $[0,1,0]$ of $P K_{2}(\mathbf{Q}(\varepsilon))$ as the image of some collineations of the plane $P K_{2}(\mathbf{Q}(\varepsilon))$. To do this, we give the collineations $S_{a}$ and $L_{a}$. Later we show that the addition and multiplication of the nonneighbor points on the line $[0,1,0]$ can be obtained as the images under that $S_{a}$ and $L_{a}$.
\end{abstract}

MSC: 51C05; 51 J10; $12 \mathrm{E} 15$

Keywords: projective Klingenberg planes; collineations; local ring; division ring

\section{Introduction and preliminaries}

In the plane geometry, there are three important classes: affine planes, projective planes and hyperbolic planes. In recent years, studies on the generalization of these classes are becoming more popular. In this paper, we study on the projective Klingenberg planes, which are generalizations of the projective planes. Now we give some required concepts from [14] for understanding projective Klingenberg planes. A ring $\mathbf{R}:=(\mathbf{R},+, \cdot)$ is defined as a set $\mathbf{R}$ together with two binary operations + and ., which we call addition and multiplication, such that the following axioms are satisfied:

$\mathrm{R} 1:(\mathbf{R},+)$ is an Abelian group;

R2: Multiplication is associative;

R3: Distributive laws holds.

A ring $\mathbf{R}$ with identity element is called local if the set $\mathbf{I}$ of its non-units forms an ideal.

A Projective Plane $\Pi=(\mathcal{P}, \mathcal{L}, \in)$ is a system in which the elements of $\mathcal{P}$ are called points and the elements of $\mathcal{L}$ are called lines together with an incidence relation $\in$ between the points and lines such that

P1: If $P$ and $Q$ are distinct points, then there is a unique line passing through $P$ and $Q$ (denoted by $P \vee Q$ or $P Q$ );

P2: If $l$ and $m$ are any lines, then there exist at least one point on both $l$ and $m$;

P3: There exists four points such that no three of them are collinear.

In any projective plane, it is well known that there is a unique point on any distinct line pair and if $l$ and $m$ are distinct lines, the intersection point of these lines is denoted by $l \wedge m$ or $l m$.

๑) 2013 Celik and Dayioglu; licensee Springer. This is an Open Access article distributed under the terms of the Creative Commons Attribution License (http://creativecommons.org/licenses/by/2.0), which permits unrestricted use, distribution, and reproduction in any medium, provided the original work is properly cited. 
A Projective Klingenberg plane (PK-Plane) is a system $(\mathcal{P}, \mathcal{L}, \in, \sim)$ where $(\mathcal{P}, \mathcal{L}, \in)$ is an incidence structure and $\sim$ is an equivalence relation on $\mathcal{P} \cup \mathcal{L}$ (called neighboring) such that no point is neighbor to any line and the following axioms are satisfied:

PK1: If $P$ and $Q$ are non-neighbor points, then there is a unique line passing through $P$ and $Q$;

PK2: If $l$ and $m$ are non-neighbor lines, then there is a unique point on both $l$ and $m$;

PK3: There is a projective plane $\Pi^{*}$ and an incidence structure epimorphism

$$
\chi: \Pi \longrightarrow \Pi^{*} \text { such that } P \sim Q \Longleftrightarrow \chi(P)=\chi(Q) \text { and } l \sim m \Longleftrightarrow \chi(l)=\chi(m) .
$$

A point $P \in \mathcal{P}$ is called near a line $g \in \mathcal{L}$ (which is denoted by $P \sim g$ ) iff there exists a line $h \sim g$ such that $P \in h$.

An incidence structure automorphism preserving and reflecting the neighbor relation is called a collineation of $\Pi$.

Let $\Pi$ be a PK-plane with canonical image $\Pi^{*}$. Choose a basis $(O, U, V, E)$ whose image $(\chi(O), \chi(U), \chi(V), \chi(E))$ in $\Pi^{*}$ form a quadrangle. Let $g_{\infty}:=U V, l:=O E, W:=l \wedge(U V)$, $\eta:=\{P \in l \mid P \sim O\}$ and $R:=\{P \in l \mid P \nsim W\}$. Let $0:=O, 1:=E$. Then the points $P \in \mathcal{P}$ and the lines $g \in \mathcal{L}$ of $\Pi$ get their coordinates as follows:

If $P \nsim g_{\infty}$, let $P=(x, y, 1)$ where $(x, x, 1)=(P V) \wedge l,(y, y, 1)=(P U) \wedge l$;

If $P \sim g_{\infty}, P \nsim V$ let $P=(1, y, z)$ where $(1, z, 1)=((P V \wedge U E) \vee O) \wedge E V$ and $(1, y, 1)=$ $O P \wedge E V$

If $P \sim V$, let $P=(w, 1, z)$ where $(1,1, z)=P U \wedge l$, and $(w, 1,1)=O P \wedge E U$ (clearly $w, z \in \eta)$;

If $g \nsim V$, then $g=[m, 1, k]$ where $(1, m, 1)=\left(\left(g \wedge g_{\infty}\right) \vee O\right) \wedge E V,(0, k, 1)=g \wedge O V$;

If $g \sim V, g \nsim g_{\infty}$, then $g=[1, n, p]$ where $(n, 1,1)=\left(\left(g \wedge g_{\infty}\right) \vee O\right) \wedge E U,(p, 0,1)=g \wedge O U$;

If $g \sim g_{\infty}$, then $g=[q, n, 1]$ where $(1,0, q)=g \wedge O U,(0,1, n)=g \wedge O V($ then $q, n \in \eta)$.

Then $O=(0,0,1), U=(1,0,0), V=(0,1,0), E=(1,1,1), O U=[0,1,0], O V=[1,0,0]$, $U V=[0,0,1], l=O E=[1,1,0]$ and a point $a \in \mathbf{R}$ has coordinates $(a, a, 1)$. We note that $\left(a_{1}, a_{2}, a_{3}\right) \sim\left(b_{1}, b_{2}, b_{3}\right)$ if and only if $a_{i}-b_{i} \in \mathbf{I}$, for $i=1,2,3$, dually for lines.

Let $\mathbf{R}$ be a local ring and the set of the non-units is denoted by $\mathbf{I}$. Now we recall a theorem and corollary which are constructed in [2] for Moufang-Klingenberg planes.

Theorem 1.1 The system $(\mathcal{P}, \mathcal{L}, \in, \sim)$ is a PK-plane where

$$
\begin{aligned}
& \mathcal{P}=\{(x, y, 1) \mid x, y \in \mathbf{R}\} \cup\{(1, y, z) \mid y \in \mathbf{R}, z \in \mathbf{I}\} \cup\{(w, 1, z) \mid w, z \in \mathbf{I}\}, \\
& \mathcal{L}=\{[m, 1, k] \mid m, k \in \mathbf{R}\} \cup\{[1, n, p] \mid n \in \mathbf{I}, p \in \mathbf{R}\} \cup\{[q, n, 1] \mid q, n \in \mathbf{I}\}, \\
& (x, y, 1) \in[m, 1, k] \quad \Leftrightarrow \quad y=x m+k, \\
& (x, y, 1) \in[1, n, p] \quad \Leftrightarrow \quad x=y n+p, \quad(x, y, 1) \notin[q, n, 1], \\
& (1, y, z) \in[m, 1, k] \quad \Leftrightarrow \quad y=m+z k, \\
& (1, y, z) \in[q, n, 1] \quad \Leftrightarrow \quad z=q+y n, \quad(1, y, z) \notin[1, n, p], \\
& (w, 1, z) \in[1, n, p] \quad \Leftrightarrow \quad w=n+z p, \\
& (w, 1, z) \in[q, n, 1] \quad \Leftrightarrow \quad z=w q+n, \quad(w, 1, z) \notin[m, 1, k], \\
& \left(x_{1}, x_{2}, x_{3}\right) \sim\left(y_{1}, y_{2}, y_{3}\right) \quad \Leftrightarrow \quad x_{i}-y_{i} \in \mathbf{I}, \\
& {\left[a_{1}, a_{2}, a_{3}\right] \sim\left[b_{1}, b_{2}, b_{3}\right] \quad \Leftrightarrow \quad a_{i}-b_{i} \in \mathbf{I} .}
\end{aligned}
$$


Corollary 1.2 If $t \in \mathbf{I}$, then $1-t$ is a unit and, therefore,

$$
\begin{aligned}
& (x, y, 1) \nsim(1, y, z), \quad(x, y, 1) \nsim(w, 1, z), \quad(1, y, z) \nsim(w, 1, z), \\
& (w, 1, z) \sim(u, 1, t), \quad(x, y, 1) \sim(u, v, 1) \quad \Leftrightarrow \quad(x-u \in \mathbf{I}, y-v \in \mathbf{I}), \\
& (1, y, z) \sim(1, v, t) \quad \Leftrightarrow \quad y-v \in \mathbf{I} .
\end{aligned}
$$

The PK-Plane given in Theorem 1.1 is denoted by $P K_{2}(\mathbf{R})$ and is called the PK-Plane coordinatized with (the local ring) $\mathbf{R}$.

Finally we give the definition of dual quaternions, some theorems and a corollary from [5], which we use in the next section.

We consider any quaternion ring $\mathbf{Q}=\left\{x_{0}+x_{1} i+x_{2} j+x_{3} k \mid x_{0}, x_{1}, x_{2}, x_{3} \in \mathbf{F}\right\}$ over a field $\mathbf{F}$ (which is a division ring) and the set $\mathbf{Q}(\varepsilon)=\mathbf{Q}+\mathbf{Q} \varepsilon=\{a+b \varepsilon \mid a, b \in \mathbf{Q}\}$ together with the following operations:

$$
\begin{aligned}
& (a+b \varepsilon)+(c+d \varepsilon)=(a+c)+(b+d) \varepsilon, \\
& (a+b \varepsilon)(c+d \varepsilon)=a c+(a d+b c) \varepsilon,
\end{aligned}
$$

where $\varepsilon$ represents any element not in $\mathbf{Q}$.

The elements of $\mathbf{Q}(\varepsilon)$ are called as dual quaternions. Obviously, the unity of $\mathbf{Q}(\varepsilon)$ is 1 .

Theorem 1.3 The non-unit elements of $\mathbf{Q}(\varepsilon)$ are in the form $b \varepsilon$, for $b \in \mathbf{Q}$ and if $a \neq 0$, $a, b \in Q$, then $a+b \varepsilon$ is a unit and $(a+b \varepsilon)^{-1}=a^{-1}-a^{-1} b a^{-1} \varepsilon$.

Theorem 1.4 The set of non-units $\mathbf{I}=\mathbf{Q} \varepsilon=\{b \varepsilon \mid b \in \mathbf{Q}\}$ is an ideal of $\mathbf{Q}(\varepsilon)$.

\section{Corollary 1.5}

(1) $\mathbf{Q}(\varepsilon)$ is a local ring (and it is called as the dual local ring on $\mathbf{Q}$ );

(2) It is obvious from Theorem 1.1 that $P K_{2}(\mathbf{Q}(\varepsilon))=(\mathcal{P}, \mathcal{L}, \in, \sim)$ is a PK-plane and

$$
\begin{aligned}
\mathcal{P}= & \left\{\left(x_{1}+x_{2} \varepsilon, y_{1}+y_{2} \varepsilon, 1\right) \mid x_{1}, x_{2}, y_{1}, y_{2} \in \mathbf{Q}\right\} \cup\left\{\left(1, y_{1}+y_{2} \varepsilon, z_{2} \varepsilon\right) \mid y_{1}, y_{2}, z_{2} \in \mathbf{Q}\right\} \\
& \cup\left\{\left(w_{2} \varepsilon, 1, z_{2} \varepsilon\right) \mid w_{2}, z_{2} \in \mathbf{Q}\right\}, \\
\mathcal{L}= & \left\{\left[m_{1}+m_{2} \varepsilon, 1, k_{1}+k_{2} \varepsilon\right] \mid m_{1}, m_{2}, k_{1}, k_{2} \in \mathbf{Q}\right\} \\
& \cup\left\{\left[1, n_{2} \varepsilon, p_{1}+p_{2} \varepsilon\right] \mid n_{2}, p_{1}, p_{2} \in \mathbf{Q}\right\} \cup\left\{\left[q_{2} \varepsilon, n_{2} \varepsilon, 1\right] \mid q_{2}, n_{2} \in \mathbf{Q}\right\} .
\end{aligned}
$$

Theorem 1.6 Neighbor relation $\sim$ is an equivalence relation over $\mathcal{P}$ and $\mathcal{L}$ in $P K_{2}(\mathbf{Q}(\varepsilon))$.

Theorem 1.7 In $P K_{2}(\mathbf{Q}(\varepsilon))$ the following properties are satisfied:

(1) $\left(x_{1}+x_{2} \varepsilon, y_{1}+y_{2} \varepsilon, 1\right) \in\left[m_{1}+m_{2} \varepsilon, 1, k_{1}+k_{2} \varepsilon\right] \Longleftrightarrow y_{1}=x_{1} m_{1}+k_{1}$, $y_{2}=x_{2} m_{1}+x_{1} m_{2}+k_{2}$

(2) $\left(x_{1}+x_{2} \varepsilon, y_{1}+y_{2} \varepsilon, 1\right) \in\left[1, n_{2} \varepsilon, p_{1}+p_{2} \varepsilon\right] \Longleftrightarrow x_{1}=p_{1}, x_{2}=y_{1} n_{2}+p_{2}$;

(3) $\left(1, y_{1}+y_{2} \varepsilon, z_{2} \varepsilon\right) \in\left[m_{1}+m_{2} \varepsilon, 1, k_{1}+k_{2} \varepsilon\right] \Longleftrightarrow y_{1}=m_{1}, y_{2}=m_{2}+z_{2} k_{1}$;

(4) $\left(1, y_{1}+y_{2} \varepsilon, z_{2} \varepsilon\right) \in\left[q_{2} \varepsilon, n_{2} \varepsilon, 1\right] \Longleftrightarrow z_{2}=q_{2}+y_{1} n_{2}$;

(5) $\left(w_{2} \varepsilon, 1, z_{2} \varepsilon\right) \in\left[1, n_{2} \varepsilon, p_{1}+p_{2} \varepsilon\right] \Longleftrightarrow w_{2}=n_{2}+z_{2} p_{1}$;

(6) $\left(w_{2} \varepsilon, 1, z_{2} \varepsilon\right) \in\left[q_{2} \varepsilon, n_{2} \varepsilon, 1\right] \Longleftrightarrow z_{2}=n_{2}$;

(7) $\left(a_{1}+a_{2} \varepsilon, b_{1}+b_{2} \varepsilon, 1\right) \sim\left(c_{1}+c_{2} \varepsilon, d_{1}+d_{2} \varepsilon, 1\right) \Longleftrightarrow c_{1}=a_{1} \wedge d_{1}=b_{1}$; 
(8) $\left(1, a_{1}+a_{2} \varepsilon, b_{2} \varepsilon\right) \sim\left(1, c_{1}+c_{2} \varepsilon, d_{2} \varepsilon\right) \Longleftrightarrow c_{1}=a_{1}$;

(9) For every $a_{2}, b_{2}, c_{2}, d_{2} \in \mathbf{Q} ;\left(a_{2} \varepsilon, 1, b_{2} \varepsilon\right) \sim\left(c_{2} \varepsilon, 1, d_{2} \varepsilon\right)$.

\section{Two collineations of $P K_{2}(\mathrm{Q}(\varepsilon))$}

In this section, we will define two transformations for the points and lines of $P K_{2}(\mathbf{Q}(\varepsilon))$ and also we will show that these transformations are collineations. Similar transformations can be found in [6].

Let $a=a_{1}+a_{2} \varepsilon$ be an arbitrary element of $\mathbf{Q}(\varepsilon)$. Then we define a transformation $S_{a}$ : $P K_{2}(\mathbf{Q}(\varepsilon)) \longrightarrow P K_{2}(\mathbf{Q}(\varepsilon))$ as:

$$
\begin{aligned}
& \left(x_{1}+x_{2} \varepsilon, y_{1}+y_{2} \varepsilon, 1\right) \longrightarrow\left(x_{1}+a_{1}+\left(x_{2}+a_{2}\right) \varepsilon, y_{1}+y_{2} \varepsilon, 1\right), \\
& \left(1, y_{1}+y_{2} \varepsilon, z_{2} \varepsilon\right) \longrightarrow\left(1, y_{1}+\left(y_{2}-z_{2} a_{1} y_{1}\right) \varepsilon, z_{2} \varepsilon\right), \\
& \left(w_{2} \varepsilon, 1, z_{2} \varepsilon\right) \longrightarrow\left(\left(w_{2}+z_{2} a_{1}\right) \varepsilon, 1, z_{2} \varepsilon\right), \\
& {\left[m_{1}+m_{2} \varepsilon, 1, k_{1}+k_{2} \varepsilon\right] \longrightarrow\left[m_{1}+m_{2} \varepsilon, 1, k_{1}-a_{1} m_{1}+\left(k_{2}-a_{1} m_{2}-a_{2} m_{1}\right) \varepsilon\right],} \\
& {\left[1, n_{2} \varepsilon, p_{1}+p_{2} \varepsilon\right] \longrightarrow\left[1, n_{2} \varepsilon, p_{1}+a_{1}+\left(p_{2}+a_{2}\right) \varepsilon\right],} \\
& {\left[q_{2} \varepsilon, n_{2} \varepsilon, 1\right] \longrightarrow\left[q_{2} \varepsilon, n_{2} \varepsilon, 1\right] .}
\end{aligned}
$$

Similarly, we define a transformation $L_{a}: P K_{2}(\mathbf{Q}(\varepsilon)) \longrightarrow P K_{2}(\mathbf{Q}(\varepsilon))$ where $a=a_{1}+a_{2} \varepsilon \in$ $\mathbf{Q}(\varepsilon)$ and $a_{1} \neq 0$ as:

$$
\begin{aligned}
& \left(x_{1}+x_{2} \varepsilon, y_{1}+y_{2} \varepsilon, 1\right) \\
& \quad \longrightarrow\left(a_{1} x_{1}+\left(a_{1} x_{2}+a_{2} x_{1}\right) \varepsilon, a_{1} y_{1} a_{1}+\left(a_{1} y_{1} a_{2}+a_{1} y_{2} a_{1}+a_{2} y_{1} a_{1}\right) \varepsilon, 1\right), \\
& \left(1, y_{1}+y_{2} \varepsilon, z_{2} \varepsilon\right) \longrightarrow\left(1, y_{1} a_{1}+\left(y_{1} a_{2}+y_{2} a_{1}\right) \varepsilon,\left(z_{2} a_{1}^{-1}\right) \varepsilon\right), \\
& \left(w_{2} \varepsilon, 1, z_{2} \varepsilon\right) \longrightarrow\left(\left(a_{1}^{-1} w_{2}\right) \varepsilon, 1,\left(a_{1}^{-1} z_{2} a_{1}^{-1}\right) \varepsilon\right), \\
& {\left[m_{1}+m_{2} \varepsilon, 1, k_{1}+k_{2} \varepsilon\right]} \\
& \quad \longrightarrow\left[m_{1} a_{1}+\left(m_{1} a_{2}+m_{2} a_{1}\right) \varepsilon, 1, a_{1} k_{1} a_{1}+\left(a_{1} k_{1} a_{2}+a_{1} k_{2} a_{1}+a_{2} k_{1} a_{1}\right) \varepsilon\right] \\
& {\left[1, n_{2} \varepsilon, p_{1}+p_{2} \varepsilon\right] \longrightarrow\left[1,\left(a_{1}^{-1} n_{2}\right) \varepsilon, a_{1} p_{1}+\left(a_{1} p_{2}+a_{2} p_{1}\right) \varepsilon\right],} \\
& {\left[q_{2} \varepsilon, n_{2} \varepsilon, 1\right] \longrightarrow\left[\left(q_{2} a_{1}^{-1}\right) \varepsilon,\left(a_{1}^{-1} n_{2} a_{1}^{-1}\right) \varepsilon, 1\right] .}
\end{aligned}
$$

Now, we can give the following theorem.

Theorem 2.1 The transformations $S_{a}$ and $L_{a}$ defined above are collineations of $P K_{2}(\mathbf{Q}(\varepsilon))$.

Proof It must be shown that $S_{a}$ and $L_{a}$ are bijective and preserves the incidence and the neighbor relations.

It can be shown that $S_{a}$ and $L_{a}$ are one.to.one transformations. Also since;

$$
\begin{aligned}
& S_{a}\left(x_{1}-a_{1}+\left(x_{2}-a_{2}\right) \varepsilon, y_{1}+y_{2} \varepsilon, 1\right)=\left(x_{1}+x_{2} \varepsilon, y_{1}+y_{2} \varepsilon, 1\right), \\
& S_{a}\left(1, y_{1}+\left(y_{2}+z_{2} a_{1} y_{1}\right) \varepsilon, z_{2} \varepsilon\right)=\left(1, y_{1}+y_{2} \varepsilon, z_{2} \varepsilon\right), \\
& S_{a}\left(\left(w_{2}-z_{2} a_{1}\right) \varepsilon, 1, z_{2} \varepsilon\right)=\left(w_{2} \varepsilon, 1, z_{2} \varepsilon\right), \\
& S_{a}\left[m_{1}+m_{2} \varepsilon, 1, k_{1}+a_{1} m_{1}+\left(k_{2}+a_{1} m_{2}+a_{2} m_{1}\right) \varepsilon\right]=\left[m_{1}+m_{2} \varepsilon, 1, k_{1}+k_{2} \varepsilon\right],
\end{aligned}
$$




$$
\begin{aligned}
& S_{a}\left[1, n_{2} \varepsilon, p_{1}-a_{1}+\left(p_{2}-a_{2}\right) \varepsilon\right]=\left[1, n_{2} \varepsilon, p_{1}+p_{2} \varepsilon\right], \\
& S_{a}\left[q_{2} \varepsilon, n_{2} \varepsilon, 1\right]=\left[q_{2} \varepsilon, n_{2} \varepsilon, 1\right]
\end{aligned}
$$

and

$$
\begin{aligned}
& L_{a}\left(a_{1}^{-1} x_{1}+\left(a_{1}^{-1}\left(x_{2}-a_{2} a_{1}^{-1} x_{1}\right)\right) \varepsilon, a_{1}^{-1} y_{1} a_{1}^{-1}+\left(a_{1}^{-1}\left(\left(y_{2}-y_{1} a_{1}^{-1} a_{2}\right) a_{1}^{-1}-a_{2} a_{1}^{-1} y_{1} a_{1}^{-1}\right) \varepsilon, 1\right)\right) \\
& \quad \quad=\left(x_{1}+x_{2} \varepsilon, y_{1}+y_{2} \varepsilon, 1\right) \\
& L_{a}\left(1, y_{1} a_{1}^{-1}+\left(\left(y_{2}-y_{1} a_{1}^{-1} a_{2}\right) a_{1}^{-1}\right) \varepsilon,\left(z_{2} a_{1}\right) \varepsilon\right)=\left(1, y_{1}+y_{2} \varepsilon, z_{2} \varepsilon\right) \\
& L_{a}\left(\left(a_{1} w_{2}\right) \varepsilon, 1,\left(a_{1} z_{2} a_{1}\right) \varepsilon\right)=\left(w_{2} \varepsilon, 1, z_{2} \varepsilon\right) \\
& L_{a}\left[a_{1}^{-1} m_{1}+\left(a_{1}^{-1}\left(m_{2}-a_{2} a_{1}^{-1} m_{1}\right)\right) \varepsilon, 1, a_{1}^{-1} k_{1} a_{1}^{-1}+\left(a_{1}^{-1}\left(k_{2}-k_{1} a_{1}^{-1} a_{2}-a_{2} a_{1}^{-1} k_{1}\right) a_{1}^{-1}\right) \varepsilon\right] \\
& \quad=\left[m_{1}+m_{2} \varepsilon, 1, k_{1}+k_{2} \varepsilon\right] \\
& L_{a}\left[1,\left(a_{1} n_{2}\right) \varepsilon, a_{1}^{-1} p_{1}+\left(a_{1}^{-1}\left(p_{2}-a_{2} a_{1}^{-1} p_{1}\right)\right) \varepsilon\right]=\left[1, n_{2} \varepsilon, p_{1}+p_{2} \varepsilon\right] \\
& L_{a}\left[\left(q_{2} a_{1}\right) \varepsilon,\left(a_{1} n_{2} a_{1}\right) \varepsilon, 1\right]=\left[q_{2} \varepsilon, n_{2} \varepsilon, 1\right],
\end{aligned}
$$

we find that $S_{a}$ and $L_{a}$ are surjective and also since,

$$
\begin{aligned}
& S_{a}\left(x_{1}+x_{2} \varepsilon, y_{1}+y_{2} \varepsilon, 1\right) \in S_{a}\left[m_{1}+m_{2} \varepsilon, 1, k_{1}+k_{2} \varepsilon\right] \\
& \Longleftrightarrow y_{1}+y_{2} \varepsilon=\left(x_{1}+a_{1}+\left(x_{2}+a_{2}\right) \varepsilon\right)\left(m_{1}+m_{2} \varepsilon\right)+k_{1}-a_{1} m_{1} \\
& +\left(k_{2}-a_{1} m_{2}-a_{2} m_{1}\right) \varepsilon \\
& \Longleftrightarrow y_{1}=x_{1} m_{1}+k_{1} \wedge y_{2}=x_{1} m_{2}+x_{2} m_{1}+k_{2} \\
& \Longleftrightarrow\left(x_{1}+x_{2} \varepsilon, y_{1}+y_{2} \varepsilon, 1\right) \in\left[m_{1}+m_{2} \varepsilon, 1, k_{1}+k_{2} \varepsilon\right], \\
& S_{a}\left(x_{1}+x_{2} \varepsilon, y_{1}+y_{2} \varepsilon, 1\right) \in S_{a}\left[1, n_{2} \varepsilon, p_{1}+p_{2} \varepsilon\right] \\
& \Longleftrightarrow x_{1}+a_{1}+\left(x_{2}+a_{2}\right) \varepsilon=\left(y_{1}+y_{2} \varepsilon\right)\left(n_{2} \varepsilon\right)+p_{1}+a_{1}+\left(p_{2}+a_{2}\right) \varepsilon \\
& \Longleftrightarrow \quad x_{1}=p_{1} \wedge x_{2}=y_{1} n_{2}+p_{2} \\
& \Longleftrightarrow \quad\left(x_{1}+x_{2} \varepsilon, y_{1}+y_{2} \varepsilon, 1\right) \in\left[1, n_{2} \varepsilon, p_{1}+p_{2} \varepsilon\right], \\
& \Longleftrightarrow y_{1}+\left(y_{2}-z_{2} a_{1} y_{1}\right) \varepsilon=m_{1}+m_{2} \varepsilon+\left(z_{2} \varepsilon\right)\left(k_{1}-a_{1} m_{1}+\left(k_{2}-a_{1} m_{2}-a_{2} m_{1}\right) \varepsilon\right) \\
& \Longleftrightarrow y_{1}=m_{1} \wedge y_{2}=m_{2}+z_{2} k_{1} \\
& \Longleftrightarrow \quad\left(1, y_{1}+y_{2} \varepsilon, z_{2} \varepsilon\right) \in\left[m_{1}+m_{2} \varepsilon, 1, k_{1}+k_{2} \varepsilon\right], \\
& \Longleftrightarrow \quad z_{2} \varepsilon=q_{2} \varepsilon+\left(y_{1}+\left(y_{2}-z_{2} a_{1} y_{1}\right) \varepsilon\right)\left(n_{2} \varepsilon\right) \\
& \Longleftrightarrow \quad z_{2} \varepsilon=q_{2} \varepsilon+\left(y_{1} n_{2}\right) \varepsilon \\
& \Longleftrightarrow \quad\left(1, y_{1}+y_{2} \varepsilon, z_{2} \varepsilon\right) \in\left[q_{2} \varepsilon, n_{2} \varepsilon, 1\right],
\end{aligned}
$$

$S_{a}\left(w_{2} \varepsilon, 1, z_{2} \varepsilon\right) \in S_{a}\left[1, n_{2} \varepsilon, p_{1}+p_{2} \varepsilon\right]$

$$
\Longleftrightarrow \quad\left(w_{2}+z_{2} a_{1}\right) \varepsilon=n_{2} \varepsilon+\left(z_{2} \varepsilon\right)\left(p_{1}+a_{1}+\left(p_{2}+a_{2}\right) \varepsilon\right)
$$




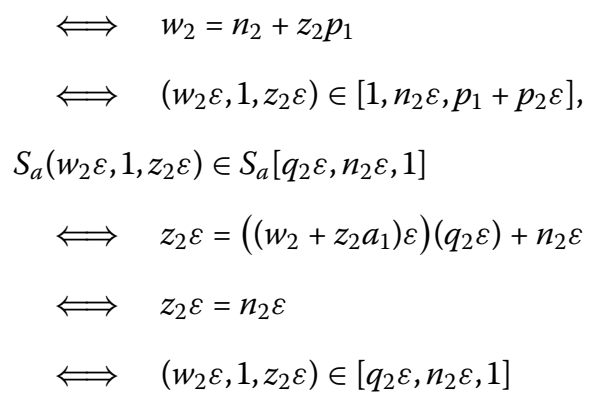

and

$$
\begin{aligned}
& L_{a}\left(x_{1}+x_{2} \varepsilon, y_{1}+y_{2} \varepsilon, 1\right) \in L_{a}\left[m_{1}+m_{2} \varepsilon, 1, k_{1}+k_{2} \varepsilon\right] \\
& \Longleftrightarrow y_{1}=x_{1} m_{1}+k_{1} \wedge y_{2}=x_{1} m_{2}+x_{2} m_{1}+k_{2} \\
& \Longleftrightarrow \quad\left(x_{1}+x_{2} \varepsilon, y_{1}+y_{2} \varepsilon, 1\right) \in\left[m_{1}+m_{2} \varepsilon, 1, k_{1}+k_{2} \varepsilon\right], \\
& L_{a}\left(x_{1}+x_{2} \varepsilon, y_{1}+y_{2} \varepsilon, 1\right) \in L_{a}\left[1, n_{2} \varepsilon, p_{1}+p_{2} \varepsilon\right] \\
& \Longleftrightarrow \quad x_{1}=p_{1} \wedge x_{2}=y_{1} n_{2}+p_{2} \\
& \Longleftrightarrow \quad\left(x_{1}+x_{2} \varepsilon, y_{1}+y_{2} \varepsilon, 1\right) \in\left[1, n_{2} \varepsilon, p_{1}+p_{2} \varepsilon\right], \\
& L_{a}\left(1, y_{1}+y_{2} \varepsilon, z_{2} \varepsilon\right) \in L_{a}\left[m_{1}+m_{2} \varepsilon, 1, k_{1}+k_{2} \varepsilon\right] \\
& \Longleftrightarrow \quad y_{1}=m_{1} \wedge y_{2}=m_{2}+z_{2} k_{1} \\
& \Longleftrightarrow \quad\left(1, y_{1}+y_{2} \varepsilon, z_{2} \varepsilon\right) \in\left[m_{1}+m_{2} \varepsilon, 1, k_{1}+k_{2} \varepsilon\right], \\
& L_{a}\left(1, y_{1}+y_{2} \varepsilon, z_{2} \varepsilon\right) \in L_{a}\left[q_{2} \varepsilon, n_{2} \varepsilon, 1\right] \\
& \Longleftrightarrow \quad z_{2}=q_{2}+y_{1} n_{2} \\
& \Longleftrightarrow \quad\left(1, y_{1}+y_{2} \varepsilon, z_{2} \varepsilon\right) \in\left[q_{2} \varepsilon, n_{2} \varepsilon, 1\right] \text {, } \\
& L_{a}\left(w_{2} \varepsilon, 1, z_{2} \varepsilon\right) \in L_{a}\left[1, n_{2} \varepsilon, p_{1}+p_{2} \varepsilon\right] \\
& \Longleftrightarrow \quad w_{2}=n_{2}+z_{2} p_{1} \\
& \Longleftrightarrow \quad\left(w_{2} \varepsilon, 1, z_{2} \varepsilon\right) \in\left[1, n_{2} \varepsilon, p_{1}+p_{2} \varepsilon\right], \\
& L_{a}\left(w_{2} \varepsilon, 1, z_{2} \varepsilon\right) \in L_{a}\left[q_{2} \varepsilon, n_{2} \varepsilon, 1\right] \\
& \Longleftrightarrow \quad z_{2} \varepsilon=n_{2} \varepsilon \\
& \Longleftrightarrow \quad\left(w_{2} \varepsilon, 1, z_{2} \varepsilon\right) \in\left[q_{2} \varepsilon, n_{2} \varepsilon, 1\right],
\end{aligned}
$$

we have that $S_{a}$ and $L_{a}$ preserves the incidence relation. Finally, since

$$
\begin{aligned}
& S_{a}\left(x_{1}+x_{2} \varepsilon, y_{1}+y_{2} \varepsilon, 1\right) \sim S_{a}\left(u_{1}+u_{2} \varepsilon, v_{1}+v_{2} \varepsilon, 1\right) \\
& \quad \Longleftrightarrow \quad x_{1}-u_{1}=0 \wedge y_{1}-v_{1}=0 \\
& \quad \Longleftrightarrow \quad\left(x_{1}+x_{2} \varepsilon, y_{1}+y_{2} \varepsilon, 1\right) \sim\left(u_{1}+u_{2} \varepsilon, v_{1}+v_{2} \varepsilon, 1\right), \\
& S_{a}\left(1, y_{1}+y_{2} \varepsilon, z_{2} \varepsilon\right) \sim S_{a}\left(1, v_{1}+v_{2} \varepsilon, t_{2} \varepsilon\right) \\
& \quad \Longleftrightarrow \quad y_{1}-v_{1}=0
\end{aligned}
$$




$$
\begin{gathered}
\Longleftrightarrow \quad\left(1, y_{1}+y_{2} \varepsilon, z_{2} \varepsilon\right) \sim\left(1, v_{1}+v_{2} \varepsilon, t_{2} \varepsilon\right), \\
S_{a}\left(w_{2} \varepsilon, 1, z_{2} \varepsilon\right) \sim S_{a}\left(u_{2} \varepsilon, 1, t_{2} \varepsilon\right) \\
\Longleftrightarrow \quad\left(w_{2} \varepsilon, 1, z_{2} \varepsilon\right) \sim\left(u_{2} \varepsilon, 1, t_{2} \varepsilon\right), \\
S_{a}\left[m_{1}+m_{2} \varepsilon, 1, k_{1}+k_{2} \varepsilon\right] \sim S_{a}\left[u_{1}+u_{2} \varepsilon, 1, t_{1}+t_{2} \varepsilon\right] \\
\Longleftrightarrow \quad m_{1}-u_{1}=0 \wedge k_{1}-t_{1}=0 \\
\quad \Longleftrightarrow \quad\left[m_{1}+m_{2} \varepsilon, 1, k_{1}+k_{2} \varepsilon\right] \sim\left[u_{1}+u_{2} \varepsilon, 1, t_{1}+t_{2} \varepsilon\right], \\
S_{a}\left[1, n_{2} \varepsilon, p_{1}+p_{2} \varepsilon\right] \sim S_{a}\left[1, v_{2} \varepsilon, t_{1}+t_{2} \varepsilon\right] \\
\Longleftrightarrow \quad p_{1}-t_{1}=0 \\
\quad \Longleftrightarrow \quad\left[1, n_{2} \varepsilon, p_{1}+p_{2} \varepsilon\right] \sim\left[1, v_{2} \varepsilon, t_{1}+t_{2} \varepsilon\right], \\
S_{a}\left[q_{2} \varepsilon, n_{2} \varepsilon, 1\right] \sim S_{a}\left[u_{2} \varepsilon, v_{2} \varepsilon, 1\right] \\
\quad \Longleftrightarrow \quad\left[q_{2} \varepsilon, n_{2} \varepsilon, 1\right] \sim\left[u_{2} \varepsilon, v_{2} \varepsilon, 1\right]
\end{gathered}
$$

and

$$
\begin{aligned}
& L_{a}\left(x_{1}+x_{2} \varepsilon, y_{1}+y_{2} \varepsilon, 1\right) \sim L_{a}\left(u_{1}+u_{2} \varepsilon, v_{1}+v_{2} \varepsilon, 1\right) \\
& \Longleftrightarrow x_{1}-u_{1}=0 \wedge y_{1}-v_{1}=0 \\
& \Longleftrightarrow\left(x_{1}+x_{2} \varepsilon, y_{1}+y_{2} \varepsilon, 1\right) \sim\left(u_{1}+u_{2} \varepsilon, v_{1}+v_{2} \varepsilon, 1\right) \text {, } \\
& L_{a}\left(1, y_{1}+y_{2} \varepsilon, z_{2} \varepsilon\right) \sim L_{a}\left(1, v_{1}+v_{2} \varepsilon, t_{2} \varepsilon\right) \\
& \Longleftrightarrow y_{1}-v_{1}=0 \\
& \Longleftrightarrow \quad\left(1, y_{1}+y_{2} \varepsilon, z_{2} \varepsilon\right) \sim\left(1, v_{1}+v_{2} \varepsilon, t_{2} \varepsilon\right), \\
& L_{a}\left(w_{2} \varepsilon, 1, z_{2} \varepsilon\right) \sim L_{a}\left(u_{2} \varepsilon, 1, t_{2} \varepsilon\right) \\
& \Longleftrightarrow \quad\left(w_{2} \varepsilon, 1, z_{2} \varepsilon\right) \sim\left(u_{2} \varepsilon, 1, t_{2} \varepsilon\right), \\
& L_{a}\left[m_{1}+m_{2} \varepsilon, 1, k_{1}+k_{2} \varepsilon\right] \sim L_{a}\left[u_{1}+u_{2} \varepsilon, 1, t_{1}+t_{2} \varepsilon\right] \\
& \Longleftrightarrow \quad m_{1}-u_{1}=0 \wedge k_{1}-t_{1}=0 \\
& \Longleftrightarrow \quad\left[m_{1}+m_{2} \varepsilon, 1, k_{1}+k_{2} \varepsilon\right] \sim\left[u_{1}+u_{2} \varepsilon, 1, t_{1}+t_{2} \varepsilon\right], \\
& L_{a}\left[1, n_{2} \varepsilon, p_{1}+p_{2} \varepsilon\right] \sim L_{a}\left[1, v_{2} \varepsilon, t_{1}+t_{2} \varepsilon\right] \\
& \Longleftrightarrow p_{1}-t_{1}=0 \\
& \Longleftrightarrow \quad\left[1, n_{2} \varepsilon, p_{1}+p_{2} \varepsilon\right] \sim\left[1, v_{2} \varepsilon, t_{1}+t_{2} \varepsilon\right], \\
& L_{a}\left[q_{2} \varepsilon, n_{2} \varepsilon, 1\right] \sim L_{a}\left[u_{2} \varepsilon, v_{2} \varepsilon, 1\right] \\
& \Longleftrightarrow \quad\left[q_{2} \varepsilon, n_{2} \varepsilon, 1\right] \sim\left[u_{2} \varepsilon, v_{2} \varepsilon, 1\right],
\end{aligned}
$$

we conclude that $S_{a}$ and $L_{a}$ preserves the neighbor relation.

\section{Addition and multiplication of points and their correspondences with collineations}

In this section, we recall some definitions, theorems and results about geometric addition and multiplication of points on $\mathrm{OU}$ in $\mathrm{PK}_{2}(\mathbf{Q}(\varepsilon))$ from [7] and also we will determine same 
relations between $S_{a}, L_{a}$ and geometric definitions of addition and multiplication of points on $O U$ where $(O, U, V, E)$ is a base of $P K_{2}(\mathbf{Q}(\varepsilon))$.

Definition 3.1 Let $A$ and $B$ be non-neighbor points of $P K_{2}(\mathbf{Q}(\varepsilon))$ on the line $O U$. Then

(1) $A+B$ is defined as the intersection point of the lines $L V$ and $O U$ where $L=K U \wedge B S, K=A V \wedge O S, S=(1,1,0)$;

(2) $A \cdot B$ is defined as the intersection point of the lines $V N$ and $O U$ where $N=A S \wedge O M, M=B V \wedge 1 S, S=(1,1,0), 1=(1,0,1)$.

Theorem 3.2 Let $A=\left(a_{1}+a_{2} \varepsilon, 0,1\right)$ and $B=\left(b_{1}+b_{2} \varepsilon, 0,1\right)$ be non-neighbor points on the line $O U$ and $Z=\left(1,0, z_{2} \varepsilon\right)$ be the point on the line $O U$ (neighbor to $U$ ) then;

(1) $A+B=\left(\left(a_{1}+b_{1}\right)+\left(a_{2}+b_{2}\right) \varepsilon, 0,1\right)$;

(2) $A+Z=\left(1,0, z_{2} \varepsilon\right)$

(3) $A \cdot B=\left(a_{1} b_{1}+\left(a_{1} b_{2}+a_{2} b_{1}\right) \varepsilon, 0,1\right)$;

(4) $A \cdot Z=\left(1,0,\left(z_{2} a_{1}^{-1}\right) \varepsilon\right)$ where $A \nsim O$;

(5) $Z \cdot A=\left(1,0,\left(a_{1}^{-1} z_{2}\right) \varepsilon\right)$ where $A \nsim O$.

Corollary 3.3 Following statements are valid where the points $A, B, Z, O$ are defined as in Theorem 3.2 and $Y$ is a point neighbor to $(0,0,1)$ (i.e. $\left.Y \in\left\{\left(y_{2} \varepsilon, 0,1\right) \mid x_{2} \in \mathbf{Q}\right\}\right)$

(1) $A+B=B+A$ and $A+Z=Z+A$;

(2) $A+O=A$ and $O+Z=Z$;

(3) $A+Y \sim A$;

(4) $A \cdot B \neq B \cdot A$;

(5) $O \cdot A=A \cdot O=O$;

(6) $1 \cdot A=A=A \cdot 1$ and $1 \cdot Z=Z=Z \cdot 1$;

(7) $A \cdot Y \sim Y$ and $Y \cdot A \sim Y$.

Now we give a theorem which interprets the relation between the geometric addition and multiplication of points and the collineations $S_{a}, L_{a}$ which are given in last section.

Theorem 3.4 Following equalities are valid for the point $A=(a, 0,1)$ and any point $X$ on the line $\mathrm{OU}=[0,1,0]$ where $a=a_{1}+a_{2} \varepsilon \in \mathbf{Q}(\varepsilon)$ :

(1) $S_{a}(X)=X+A$;

(2) $L_{a}(X)=A \cdot X$ where $a_{1} \neq 0$.

Proof (1) If $X$ is any non-neighbor point to $U$ on the line $O U$, then there exist a $b_{1}+b_{2} \varepsilon \in$ $\mathbf{Q}(\varepsilon)$ such that $X=\left(b_{1}+b_{2} \varepsilon, 0,1\right)$. In this case,

$$
S_{a}(X)=\left(b_{1}+a_{1}+\left(b_{2}+a_{2}\right) \varepsilon, 0,1\right)=X+A .
$$

If $X$ is any point on the line $O U$ neighbor to $U$, then there exist a $z_{2} \varepsilon \in \mathbf{Q}(\varepsilon)$ such that $X=\left(1,0, z_{2} \varepsilon\right)$. In this case,

$$
S_{a}(X)=\left(1,0+\left(0-z_{2}\left(a_{1} 0\right)\right) \varepsilon, z_{2} \varepsilon\right)=\left(1,0, z_{2} \varepsilon\right)=X+A .
$$

(2) If $X$ is any non-neighbor point to $U$ on the line $O U$ then there exist a $b_{1}+b_{2} \varepsilon \in \mathbf{Q}(\varepsilon)$ such that $X=\left(b_{1}+b_{2} \varepsilon, 0,1\right)$. In this case

$$
\begin{aligned}
L_{a}(X) & =\left(a_{1} b_{1}+\left(a_{1} b_{2}+a_{2} b_{1}\right) \varepsilon, a_{1} 0 a_{1}+\left(a_{1} 0 a_{2}+a_{1} 0 a_{1}+a_{2} 0 a_{1}\right) \varepsilon, 1\right) \\
& =\left(a_{1} b_{1}+\left(a_{1} b_{2}+a_{2} b_{1}\right) \varepsilon, 0,1\right)=A \cdot X .
\end{aligned}
$$


If $X$ is any point on the line $O U$ neighbor to $U$, then there exist a $z_{2} \varepsilon \in \mathbf{Q}(\varepsilon)$ such that $X=\left(1,0, z_{2} \varepsilon\right)$. In this case,

$$
L_{a}(X)=\left(1,0 a_{1}+\left(0 a_{2}+0 a_{1}\right) \varepsilon,\left(z_{2} a_{1}^{-1}\right) \varepsilon\right)=\left(1,0,\left(z_{2} a_{1}^{-1}\right) \varepsilon\right)=A \cdot X .
$$

Therefore, $S_{a}(X)=X+A$ and $L_{a}(X)=A \cdot X$ for any point $X$ on $[0,1,0]$ where $A=(a, 0,1)$.

\section{Competing interests}

The authors declare that they have no competing interests.

\section{Authors' contributions}

Both authors have contributed equally to this paper. Both authors read and approved the final manuscript.

\section{Acknowledgements}

Dedicated to Professor Hari M Srivastava.

This work was supported by the Commission of Scientific Research Projects of Uludag University, Project number UAP(F)-2012/23.

Received: 11 January 2013 Accepted: 5 April 2013 Published: 19 April 2013

\section{References}

1. Akpinar, A, Celik, B, Ciftçi, S: Cross-ratio and 6-figures in some Moufang-Klingenberg planes. Bull. Belg. Math. Soc. Simon Stevin 15, 49-64 (2008)

2. Baker, CA, Lane, ND, Lorimer, JW: A coordinatization for Moufang-Klingenberg planes. Simon Stevin 65, 3-22 (1991)

3. Keppens, D: Coordinazation of projective Klingenberg planes. Simon Stevin 62, 63-90 (1988)

4. Jacobson, N: Lectures in Abstract Algebra, vol. 3, 3rd edn. Springer, New York (1980)

5. Dayioglu, A, Celik, B: Projective Klingenberg planes constructed with dual local rings. AIP Conf. Proc. 1389, 308-311 (2011)

6. Celik, B, Akpinar, A, Ciftci, S: 4-transitivity and 6-figures in some Moufang-Klingenberg planes. Monatshefte Math. 152, 283-294 (2007)

7. Celik, B, Erdogan, FO: On the addition and multiplication of the points in a certain class of projective Klingenberg plane. In: International Congress in Honour of Professor Hari M. Srivastava at the Auditorium at the Campus of Uludag University, Bursa-Turkey August 23-26, 2012

doi:10.1186/1029-242X-2013-193

Cite this article as: Celik and Dayioglu: The collineations which act as addition and multiplication on points in a certain class of projective Klingenberg planes. Journal of Inequalities and Applications 2013 2013:193.

\section{Submit your manuscript to a SpringerOpen ${ }^{0}$ journal and benefit from:}

- Convenient online submission

- Rigorous peer review

- Immediate publication on acceptance

Open access: articles freely available online

- High visibility within the field

- Retaining the copyright to your article 